\title{
Postoperative urinary retention in colorectal surgery within an enhanced recovery pathway
}

\author{
Fabian Grass, $\mathrm{MD},{ }^{a}$ Juliette Slieker, $\mathrm{MD},{ }^{a}$ Pierre Frauche, $\mathrm{MD},{ }^{a}$ \\ Josep Solà, PhD, ${ }^{b}$ Catherine Blanc, $M D,{ }^{c}$ Nicolas Demartines, $M D,{ }^{a, *}$ and \\ Martin Hübner, $\mathrm{MD}^{a}$ \\ ${ }^{a}$ Department of Visceral Surgery, University Hospital of Lausanne (CHUV), Lausanne, Switzerland \\ ${ }^{\mathrm{b}}$ Centre Suisse d'Electronique et de Microtechnique CSEM, Neuchâtel, Switzerland \\ ${ }^{\mathrm{c}}$ Department of Anesthesiology, University Hospital of Lausanne (CHUV), Lausanne, Switzerland
}

\section{A R T I C L E I N F O}

Article history:

Received 7 June 2016

Received in revised form

15 August 2016

Accepted 26 August 2016

Available online 2 September 2016

Keywords:

Enhanced recovery

Fast track

Colorectal

Urinary retention

\begin{abstract}
A B S T R A C T
Background: Enhanced recovery after surgery (ERAS) guidelines for colorectal surgery suggest routine transurethral bladder drainage with early removal to prevent urinary tract infection (UTI). The aim of this study was to identify risk factors for urinary retention (UR). Methods: This retrospective analysis included all colorectal patients since ERAS implementation in May 2011-November 2014. From the prospective ERAS database, over 100 items related to demographics, surgery, compliance, and outcome were analyzed. Risk factors for UR were identified by multiple logistic regressions; then, UR was correlated to functional outcomes and UTI and acute kidney injury rates.

Results: The study cohort consisted of 513 consecutive patients. Of these, 73 patients (14\%) presented with UR. Multivariate analysis identified male gender (odds ratio $1.4 ; 95 \% \mathrm{CI}$, 1-1.8; $P=0.045$ ) and postoperative thoracic epidural analgesia (EDA; odds ratio 2.6; $95 \% \mathrm{CI}$, $1.6-4.3 ; P \leq 0.001)$ as independent risk factors for postoperative UR. Functional recovery was impeded in patients with UR, who were less mobile (mobilization day $1>4 \mathrm{~h}$ : 57\% versus $70 \%, P=0.024)$ and gained more weight $(2.8 \pm 2.5 \mathrm{~kg}$ versus $1.6 \pm 3 \mathrm{~kg}$ on day $1, P=0.001)$ due to fluid overload. Furthermore, patients with urinary catheters reported more pain (visual analog scales day 3 : $3.1 \pm 2.5$ versus $2.2 \pm 2.4, P=0.002$ ) and depended longer on intravenous fluid administration (termination of intravenous fluids later than day 1: $53 \%$ versus $39 \%, P=0.021)$. Ten of 73 patients (14\%) developed UTI in patients with UR and 42 of 440 $(10 \%)$ in patients without UR $(P=0.276)$. Six of 73 patients $(8 \%)$ developed acute kidney injury in patients with UR and 36 of $440(8 \%)$ in patients without UR $(P=0.991)$.

Conclusions: Male gender and EDA were independent risk factors for postoperative UR which appeared to be a significant impediment for functional recovery.
\end{abstract}

(c) 2016 Elsevier Inc. All rights reserved.

\footnotetext{
This study was presented at the Annual Swiss Surgical Meeting 2015, May 20-22, Bern, Switzerland.

* Corresponding author. Department of Visceral Surgery, University Hospital CHUV, Bugnon 46, 1011 Lausanne, Switzerland. Tel.: +41 21 31424 00; fax: +41213142411.

E-mail address: demartines@chuv.ch (N. Demartines). 0022-4804/\$ - see front matter (c) 2016 Elsevier Inc. All rights reserved.
} 


\section{Introduction}

Enhanced recovery after surgery (ERAS) pathways in colorectal surgery have been associated with decreased complication rates, positively influencing length of stay and costs..$^{1,2}$ To promote early mobilization and thus functional recovery, adjuncts such as nasogastric tubes or postoperative urinary catheters should be avoided if possible. Hence, the ERAS guidelines suggest early postoperative removal of transurethral routine bladder drainage, regardless of the usage of thoracic epidural analgesia (EDA). ${ }^{2}$ Furthermore, it has been repeatedly demonstrated that early removal of bladder catheters decreases the rate of urinary tract infections (UTIs). ${ }^{3}$

The aim of the current study was to assess incidence and risk factors for urinary retention (UR) in a consecutive cohort of colorectal patients treated according to the ERAS pathway. In a second step, impairment of functional recovery with repetitive urinary drainage was evaluated.

\section{Methods}

The study cohort consisted of all consecutively operated colorectal surgical interventions between May 2011 and November 2014 at the University Hospital of Lausanne (CHUV). All colorectal patients were treated according to the ERAS protocol, and no patient was excluded from this retrospective analysis. Data were collected prospectively by a dedicated clinical nurse in the official ERAS Interactive Audit System. Informed consent was obtained from all patients before surgery, and the study was approved by the Institutional Review Board. The study was conducted according to the STROBE (STrengthening the Reporting of OBservational studies in Epidemiology) criteria and registered under www. researchregistry.com (UIN research registry 833).

The institutional enhanced recovery pathway was published previously ${ }^{4}$ and is in accordance with the ERAS recommendations updated $2013 .{ }^{2}$ When epidural analgesia (EDA) was considered, the catheter was inserted at a thoracic level (Th8-Th10) before induction of anesthesia, and a continuous perfusion of bupivacaine $0.5 \%$ was maintained until the end of the surgical procedure. A solution with a local anesthetic, opioids, and adrenaline (bupivacaine $0.1 \%$, fentanyl $2 \mu \mathrm{g} / \mathrm{mL}$, and adrenaline $2 \mu \mathrm{g} / \mathrm{mL}$ ) was started at the recovery room at a rate of $6-10 \mathrm{~mL} / \mathrm{h}$ and maintained for $2 \mathrm{~d}$ after minimally invasive surgery and $4 \mathrm{~d}$ after open surgery. The efficacy of EDA was evaluated every day by dedicated nurses of the analgesia unit. Patients without EDAs were managed by combinations of paracetamol, metamizole or nonsteroidal anti-inflammatory drugs, and opioids. Failure of EDA was defined as removal of EDA before the anticipated time point because of different reasons such as insufficient pain relief, dislodged or accidentally withdrawn catheters or nontolerance due to other reasons, in accordance to the descriptions of Hermanides. ${ }^{5}$ Baseline demographic and pertinent surgical information was recorded. Surgical procedures were subdivided, distinguishing further between right and left or sigmoid colectomies and rectal procedures for the purpose of this study. Extended major surgery (rectum resection, proctocolectomy, total colectomy, and ileal pouchanal anastomosis) was distinguished from major surgery (segmental colectomy, stoma procedure, and small bowel surgery). Data about ERAS-specific perioperative care items were prospectively collected. ${ }^{2}$ Urinary catheters were removed within $24 \mathrm{~h}$ after the surgical procedure, except for low rectal resections where the withdrawal of the catheter was performed on postoperative day (POD) 3 or 4. Functional recovery parameters such as mobilization, weight gain, tolerance of oral fluids or sip feeds and time to flatus and stool were assessed on a daily basis. Furthermore, patients reported on visual analog scales (VAS) the perception of pain and nausea. UTI were defined as clinical infections proved by positive urine cultures. Acute kidney injury (AKI) was defined as an increase in serum creatinine level $\times 1.5$ or decrease in glomerular filtration rate (GFR) by $25 \%{ }^{6}$

\section{Outcomes and/or study end points}

The primary end point was the rate of postoperative UR. Postoperative UR was defined as the need for postoperative insertion of a Foley catheter due to voiding problems (including single in-and-out straight catheters). Other reasons for postoperative catheter insertion such as fluid guidance or reoperation were not accounted for. Risk factors for UR were identified among demographic, surgery-related, and perioperative care items.

Secondary end points were functional recovery and urinary complications (UTI and AKI), which were compared between the two groups (UR versus no UR).

\section{Statistical analysis}

Descriptive statistics for categorical variables were reported as frequency (\%), while continuous variables were reported as mean (standard deviation) or median (interquartile range). Chi-square test was used for comparison of categorical variables. All statistical tests were two-sided, and a level of 0.05 was used to indicate statistical significance. Variables with $P$ values $\leq 0.05$ were then entered into a multivariate logistic regression (based on a probit regression model) to provide adjusted estimations of the odds ratio (OR). Data analysis was performed with the Statistical Software for the Social Sciences SPSS Advanced Statistics 22 (IBM Software Group, Chicago, IL) and MATLAB Statistical Toolbox v10.1 (Mathworks, Natick, MA).

\section{Results}

\section{Patients}

A total of 513 patients were included over the observed period. The baseline characteristics of the two groups (UR versus no UR) were comparable except for gender and World Health Organization (WHO) performance score and are illustrated in Table 1. 


\section{Table 1 - Baseline characteristics.}

\begin{tabular}{lccl} 
Item & $\begin{array}{c}\text { Urinary } \\
\text { retention } \\
(n=73)\end{array}$ & $\begin{array}{c}\text { No urinary } \\
\text { retention } \\
(n=440)\end{array}$ & P value \\
\hline Age (y) & $65.7( \pm 15.6)$ & $62.6( \pm 16.4)$ & 0.118 \\
Male gender & $47(64 \%)$ & $229(52 \%)$ & 0.05 \\
BMI $\left(\mathrm{kg} / \mathrm{m}^{2}\right)$ & $25( \pm 4.6)$ & $25.5( \pm 4.6)$ & 0.405 \\
\hline ASA group $(1-2: 3-4)$ & $57: 16$ & $320: 119$ & 0.351 \\
Smoking & $13 / 73(18 \%)$ & $81 / 402(21 \%)$ & 0.595 \\
\hline Diabetes mellitus & $8 / 73(11 \%)$ & $48 / 439(11 \%)$ & 0.995 \\
Immunosuppression & $11 / 73(15 \%)$ & $65 / 439(15 \%)$ & 0.839 \\
\hline WHO score $>2$ & $2 / 73(3 \%)$ & $43 / 436(10 \%)$ & 0.047 \\
\hline Prior radiotherapy & $9 / 73(12 \%)$ & $54 / 439(12 \%)$ & 0.995 \\
\hline Cardiac history & $27 / 73(37 \%)$ & $164 / 440(37 \%)$ & 0.963 \\
\hline Respiratory history & $8 / 73(11 \%)$ & $62 / 439(14 \%)$ & 0.466 \\
Malignancy & $32 / 73(44 \%)$ & $200 / 438(46 \%)$ & 0.772 \\
\hline
\end{tabular}

ASA = American Society of Anesthesiology; BMI = body mass index; WHO score $=$ World Health Organization performance score. Significant values are indicated in bold characters.

\section{Urinary catheter management and retention rate}

Fifty-three patients $(10 \%)$ had no urinary catheter postoperatively, and those were almost exclusively stoma procedures. Foley catheter was removed according to the protocol the morning of POD 1 in 261 patients (57\%). Urinary catheters were withdrawn later than recommended by the guidelines on POD 1 in 89 patients (32\%) after right or left colectomies. Of the 123 patients with rectal procedures, Foley catheter was removed later than on POD 1 in 81 patients (66\%).

Seventy-three patients (14\%) needed insertion of a urinary catheter because of postoperative UR. Of these, 2 (3\%) were inserted at POD 0, 38 (52\%) at POD 1, 16 (22\%) at POD 2, and 17 $(23 \%)$ at POD 3 or later. Catheters were maintained for $1 \mathrm{~d}$ or less (including in-and-out straight catheters) in 14 patients (19\%), $2 \mathrm{~d}$ in 25 patients (34\%), $3 \mathrm{~d}$ in 10 patients (14\%), and more than $3 \mathrm{~d}$ in the remaining 24 patients (33\%).

\section{Thoracic epidural anesthesia (EDA) management}

A total of 268 (52\%) had intraoperative EDA. This number dropped to 179 patients (35\%) who maintained an EDA in the postoperative period because of dysfunctioning catheters with insufficient pain relief. Of the minimally invasively operated patients, 109 (44\%) had an intraoperative EDA, compared with 159 patients (60\%) operated in an open approach $(P \leq 0.001)$. In the postoperative period, 67 patients (27\%) had EDA after laparoscopy and 112 (42\%) after laparotomy. Overall EDA failure rate due to insufficient pain relief was hence $33 \%$.

\section{Risk factors for UR}

Univariate surgical risk factors for postoperative UR were previous abdominal surgery (58\% versus $44 \%, P=0.034$ ), the duration of the operation $(230 \pm 100 \mathrm{~min}$ versus $190 \pm 100 \mathrm{~min}$, $P=0.006)$, and extended major surgery (30\% versus $17 \%$,
$P=0.008)$. Table 2 gives an overview of surgery-related items. One hundred (19\%) procedures were performed in an emergency setting. Fifty-seven percent of colorectal resections were performed minimally invasively. Left colectomies and rectum procedures had as a trend higher retention rates compared to right colectomies ( $16 \%$ versus $9 \%, P=0.096)$. Rectum procedures had as a trend higher retention rates as compared with the rest of the cohort ( $20 \%$ versus $13 \%, P=0.054)$. Among the ERAS-related items, the use of EDA, either intraoperatively ( $20 \%$ versus $8 \%, P \leq 0.001$ ) or postoperatively ( $27 \%$ versus $7 \%$, $P \leq 0.001$ ), constituted a risk factor for UR and the use of abdominal drains (23\% versus $12 \%, P=0.003)$. Figure 1 illustrates the comparison of ERAS-related items by comparing patients with UR to patients without UR. Multivariate analysis identified male gender (OR, 1.4; 95\% CI, 1-1.8, P =0.045) and postoperative EDA (OR, 2.6; 95\% CI, 1.6-4.3; $\mathrm{P} \leq 0.001)$ as independent risk factors for postoperative UR (Fig. 2).

\section{Secondary end points}

Functional recovery was slower in patients with UR, who were less mobile (mobilization at POD 1: $4.1 \pm 2.7 \mathrm{~h}$ versus $5.1 \pm 2.8 \mathrm{~h}$, $P=0.003)$ and gained more weight $(2.8 \pm 2.5 \mathrm{~kg}$ versus $1.6 \pm 3 \mathrm{~kg}$ on POD $1, P=0.001)$. Furthermore, patients with urinary catheters reported more pain (VAS score on POD 2: $3.7 \pm 2.8$ versus $2.9 \pm 2.6, P=0.01$, VAS score on POD $3: 3.1 \pm 2.5$ versus $2.2 \pm 2.4, P=0.002$ ) and depended longer on intravenous fluid administration (termination of intravenous fluids later than day 1: $53 \%$ versus $39 \% ; P=0.021$ ). Figure 3 gives an overview on different functional recovery parameters.

Fifty-two patients (10\%) developed UTI and 42 patients (8\%) developed AKI, but rates did not differ between the two groups UR versus no UR (Table 3).

\section{Discussion}

Fourteen percent of the present cohort presented with UR in the postoperative period, requiring reinsertion of a

Table 2 - Univariate surgery-related risk factors for urinary retention.

\begin{tabular}{lccc} 
Main procedure & $\begin{array}{c}\text { Urinary } \\
\text { retention } \\
(n=73), n(\%)\end{array}$ & $\begin{array}{c}\text { No urinary } \\
\text { retention }\end{array}$ & P value \\
\hline Left hemicolectomy & $19(26)$ & $125(28)$ & 0.675 \\
Right hemicolectomy & $10(14)$ & $96(22)$ & 0.113 \\
Rectum surgery & $24(33)$ & $99(23)$ & 0.054 \\
Other & $20(27)$ & $120(27)$ & 0.982 \\
Previous surgery & $42(58)$ & $194(44)$ & 0.034 \\
Minimally invasive & $26(36)$ & $220(50)$ & 0.022 \\
Emergency & $10(14)$ & $90(20)$ & 0.177 \\
Extended major surgery & $22(30)$ & $75(17)$ & 0.008 \\
Duration $>3$ h & $45(62)$ & $221(50)$ & 0.071 \\
\hline
\end{tabular}

Significant values are indicated in bold characters. 


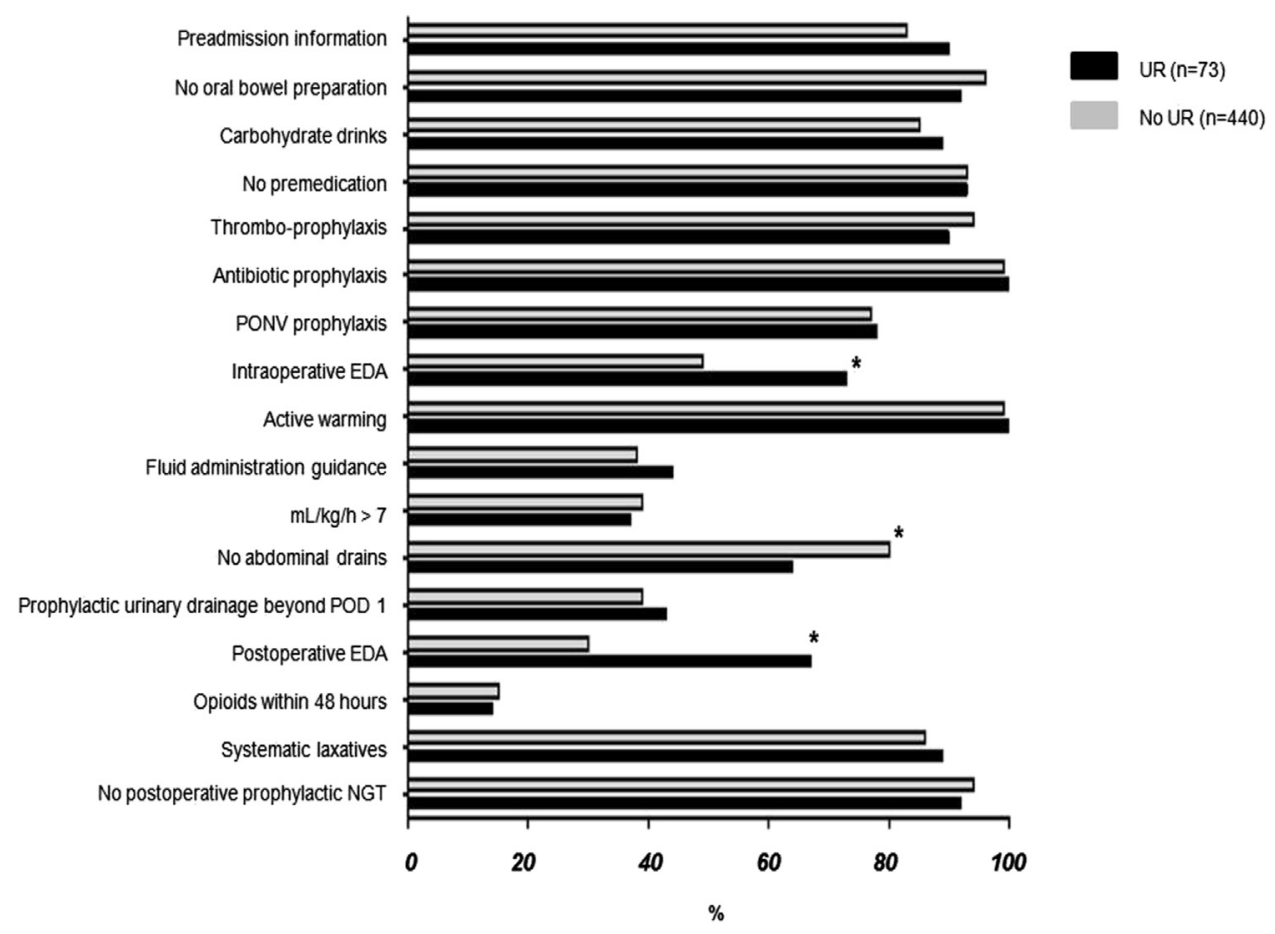

Fig. 1 - Univariate ERAS-related risk factors for UR. Comparison of different enhanced recovery after surgery-related items by comparing patients with UR (black bars) to patients without UR (gray bars). Premedication = administration of longacting sedative medication. $\mathrm{mL} / \mathrm{kg} / \mathrm{h}=$ amount of clear fluid perfusion during the surgical procedure. PONV, postoperative nausea and vomiting; NGT, nasogastric tube. *indicates statistical significance.

transurethral bladder catheter. Independent risk factors were male gender and postoperative epidurals with an almost three-fold risk for UR. UR was associated in the present cohort with slower functional recovery, as expressed by less ambulation, more pain, and more weight gain.

In major abdominal surgery, perioperative urinary catheters help to guide volume resuscitation and to maintain hemodynamic stability. ${ }^{7}$ Since adverse effects, especially UTIs, have been repeatedly associated with urinary drainage, new guidelines advocate early removal of these catheters in the postoperative period. ${ }^{2,3}$ Furthermore, Foley catheters are obvious handicaps for early mobilization. However, UR needing repetitive drainage remains a clinical reality in the surgical ward, with reported incidences ranging from $5 \%$ to $22 \%$. $^{8}$ The mechanisms that lead to UR are multifold. Injury to pelvic nerves and reflexes associated with pain lead to internal urethral sphincter contraction with consequent voiding problems after rectal surgery. ${ }^{9}$ Indeed, in the present cohort, we observed particularly high rates after rectal surgery, reaching almost $20 \%$, confirming the findings of a recently published study, which advocates at least $2 \mathrm{~d}$ of prophylactic urinary drainage to avoid UR after rectal surgery. ${ }^{10}$ This trend

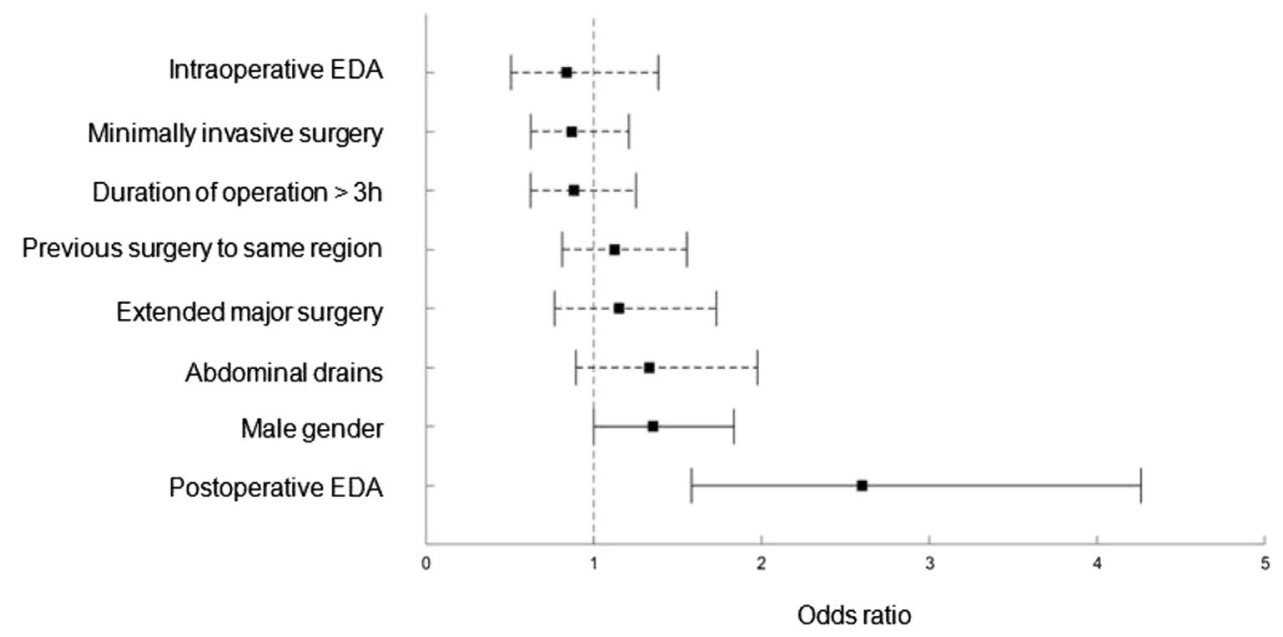

Fig. 2 - Multivariate analysis. Multivariate analysis of univariate risk factors for UR with $P<0.05$. 


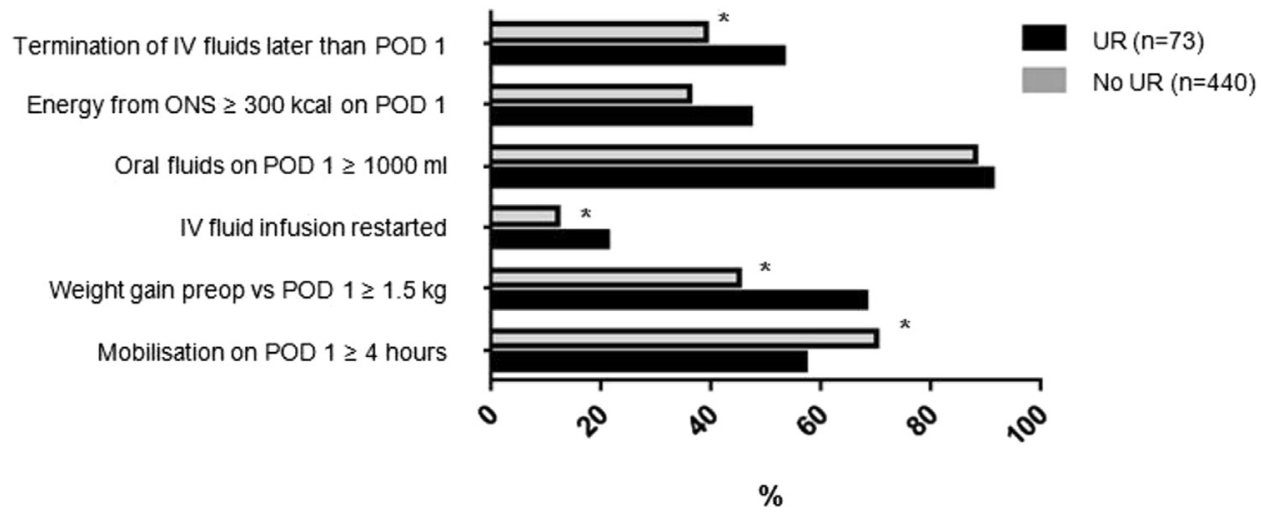

Fig. 3 - Functional recovery parameters. Comparison of functional recovery parameters by univariate analysis comparing patients with UR (black bars) with patients without UR (gray bars). ONS, oral nutritional supplement. *indicates statistical significance.

persisted when comparing rectum resections together with left colectomies to right colectomies, similar to the results of Hendren, who recommends catheter removal even on day 3-6 after rectal surgery. ${ }^{11}$ Suprapubic catheter placement has been suggested as potential alternative by a recently published systematic review that demonstrated reduced asymptomatic bacteriuria, recatheterization, and pain compared with urethral placement. ${ }^{12}$ However, for the present cohort, it has not been applied and can thus not be recommended. Further mechanisms that lead to UR have been described: overdistension of the detrusor muscle due to fluid overload and drug-associated inhibitory reflexes of the autonomic voiding complex. ${ }^{13}$ In the present cohort, UR has indeed been associated with fluid overload. However, when considering the weight and duration of the operation by calculating fluid administration in $\mathrm{mL} / \mathrm{kg} / \mathrm{h}$, we could not demonstrate any difference between the two groups, meaning that patients did in general not get excessive perioperative fluid. This reflects standardized care within the ERAS protocol, in which restrictive fluid administration is one important item.

Even if UR represents a seemingly harmless complication, disturbing consequences might result. Autonomic responses to bladder overdistension might result in pain, vomiting, hemodynamic abnormalities or even dysrhythmia. ${ }^{14}$ Furthermore, repetitive catheterization exposes the patient to urinary infections, ${ }^{3,15}$ even if this was not significant in the present cohort. Finally, permanent bladder dysfunction might lead to incontinence. ${ }^{16}$

\section{Table 3 - Secondary outcomes.}

\begin{tabular}{lccc} 
Outcome & $\begin{array}{c}\text { Urinary } \\
\text { retention } \\
(n=73), n(\%)\end{array}$ & $\begin{array}{c}\text { No urinary } \\
\text { retention }\end{array}$ & P value \\
\hline Urinary tract infection & $10(14)$ & $42(10)$ & $n(\%)$ \\
Acute kidney injury & $6(8)$ & $36(8)$ & 0.267 \\
\hline
\end{tabular}

Significant values are indicated in bold characters.
Male patients presented a two-fold higher risk of UR in the present cohort, a finding that has been described before in large multicenter trials and might reflect the occurrence of benign prostatic hyperplasia in an elderly male population. ${ }^{17}$ One way to face this problem might be perioperative tamsulosin administration, which was shown to decrease retention rates in patients undergoing pelvic surgery. ${ }^{8}$

The most important finding of the present study was the significant association of postoperative thoracic EDA and UR. In our cohort, $52 \%$ of patients had an intraoperative EDA, as advocated for open surgery by the revised ERAS guidelines. ${ }^{2}$ However, even a considerable number of minimally invasively operated patients had EDA, a concept that has been gradually reconsidered during the study period with raising new evidence. ${ }^{18} \mathrm{~A}$ recent prospective study evaluated early removal of urinary catheters within an enhanced recovery program in the presence of EDA. ${ }^{19}$ In their cohort, early removal significantly increased the risk of UR, reaching $12 \%$, similar to our observations. As our patients were treated within an enhanced recovery program, urinary catheters were withdrawn within $24 \mathrm{~h}$ of the surgical procedure according to the protocol, except for rectal resections. However, in $32 \%$ of colonic resections, urinary catheters were not withdrawn within this time frame, resulting in equally high retention rates. Since UR could repeatedly be linked to EDA, ${ }^{20}$ alternative pain treatments should be considered. ${ }^{21}$ A recently published systematic review on perioperative intravenous lidocaine infusion demonstrated promising results with pain relief especially during the first $24 \mathrm{~h}$ postoperatively. ${ }^{22}$ Ultrasound-guided transversus abdominis plane blocks on the other hand provide to some degree postoperative analgesic efficacy after abdominal laparotomy or laparoscopy, as demonstrated by the latest meta-analysis. ${ }^{23}$ Another alternative represents spinal morphine, with superior pain relief in the early postoperative period as compared with patientcontrolled morphine. ${ }^{24}$ By comparing attempted (intraoperative) and finally successful (postoperative) EDA rates, the present study observed a failure rate of sufficient analgesia of $33 \%$, which is considerable, but similar to other reported failure rates for thoracic EDA after major abdominal surgery 
between $25 \%$ and $47 \%{ }^{25,26}$ Reasons for this high failure rate were multifold: Inadequate sensitive levels or asymmetric spread, inadvertent dislodgment of the catheter, and different skill levels between senior and junior staff anesthesiologists in the setting of a teaching hospital. ${ }^{5}$

Reinsertion of a urinary catheter seemed to slow down functional recovery in our cohort, since patients expressed more pain, were less ambulant, and gained more weight compared with patients without UR. Especially within an ERAS care protocol, which demands active participation of the patient, these drawbacks impede prompt convalescence. However, a clear causative relationship between urinary catheter insertion and functional outcome cannot be established based on the presented data.

Several limitations of this present study need to be addressed. The analysis was performed retrospectively, with its inherent limitations. However, meticulous prospective documentation by an ERAS dedicated study nurse over the study period generated reliable data, and no patient was excluded from analysis. Our definition of UR was not based on clinical or radiological criteria, but objectively assessed by the rate of urinary catheter reinsertion; indication for insertion depended hence on clinical judgment.

\section{Conclusions}

UR after colorectal surgery remains a clinical problem also within enhanced recovery pathways. Postoperative insertion of urinary catheters might impede functional recovery. Since epidurals represented a major and the only modifiable risk factor for UR, alternative pain management strategies need to be considered.

\section{Acknowledgment}

The authors would like to acknowledge the support provided by all members of the Enhanced Recovery after Surgery team in Lausanne, especially Valerie Addor, dedicated clinical nurse.

Author's contributions: F.G. did design, analysis and interpretation, and drafting. J.S. helped in design, analysis and interpretation, and critical revision. P.F. and C.B. contributed to analysis and interpretation, and critical revision. J.S. did analysis and interpretation. N.D. carried out conception, interpretation, and critical revision. M.H. helped in conception and design, analysis and interpretation, and drafting. All authors approved the final version.

\section{Disclosure}

The authors have no conflicts of interest.

\section{R E F E R E N C E S}

1. Greco M, Capretti G, Beretta L, Gemma M, Pecorelli N, Braga M. Enhanced recovery program in colorectal surgery: a meta-analysis of randomized controlled trials. World J Surg. 2014;38:1531-1541.

2. Gustafsson UO, Scott MJ, Schwenk W, et al. Guidelines for perioperative care in elective colonic surgery: Enhanced Recovery after Surgery (ERAS $\left.{ }^{\circ}\right)$ Society recommendations. World J Surg. 2013;37:259-284.

3. Zaouter C, Kaneva P, Carli F. Less urinary tract infection by earlier removal of bladder catheter in surgical patients receiving thoracic epidural analgesia. Reg Anesth Pain Med. 2009;34:542-548.

4. Roulin D, Donadini A, Gander S, et al. Cost-effectiveness of the implementation of an enhanced recovery protocol for colorectal surgery. Br J Surg. 2013;100:1108-1114.

5. Hermanides J, Hollmann MW, Stevens MF, Lirk P. Failed epidural: causes and management. $\mathrm{Br} J$ Anaesth. 2012;109:144-154.

6. Bellomo R, Ronco C, Kellum JA, Mehta RL, Palevsky P, Acute Dialysis Quality Initiative w. Acute renal failure-definition, outcome measures, animal models, fluid therapy and information technology needs: the Second International Consensus Conference of the Acute Dialysis Quality Initiative (ADQI) Group. Crit Care. 2004;8:R204-R212.

7. Baldini G, Bagry H, Aprikian A, Carli F. Postoperative urinary retention: anesthetic and perioperative considerations. Anesthesiology. 2009;110:1139-1157.

8. Poylin V, Curran T, Cataldo T, Nagle D. Perioperative use of tamsulosin significantly decreases rates of urinary retention in men undergoing pelvic surgery. Int J Colorectal Dis. 2015;30:1223-1228.

9. Benoist S, Panis Y, Denet C, Mauvais F, Mariani P, Valleur P. Optimal duration of urinary drainage after rectal resection: a randomized controlled trial. Surgery. 1999;125:135-141.

10. Kwaan MR, Lee JT, Rothenberger DA, Melton GB, Madoff RD. Early removal of urinary catheters after rectal surgery is associated with increased urinary retention. Dis Colon Rectum. 2015;58:401-405.

11. Hendren S. Urinary catheter management. Clin Colon Rectal Surg. 2013;26:178-181.

12. Kidd EA, Stewart F, Kassis NC, Hom E, Omar MI. Urethral (indwelling or intermittent) or suprapubic routes for shortterm catheterisation in hospitalised adults. Cochrane Database Syst Rev. 2015;12:CD004203.

13. Keita H, Diouf E, Tubach F, et al. Predictive factors of early postoperative urinary retention in the postanesthesia care unit. Anesth Analg. 2005;101:592-596. table of contents.

14. Kamphuis ET, Ionescu TI, Kuipers PW, de Gier J, van Venrooij GE, Boon TA. Recovery of storage and emptying functions of the urinary bladder after spinal anesthesia with lidocaine and with bupivacaine in men. Anesthesiology. 1998;88:310-316.

15. Kin C, Rhoads KF, Jalali M, Shelton AA, Welton ML. Predictors of postoperative urinary retention after colorectal surgery. Dis Colon Rectum. 2013;56:738-746.

16. Mascle L, Descazeaud A, Robert G, et al. Multicenter study of Advance (R) suburethral sling for treatment of postoperative urinary incontinence of male. Prog Urol. 2015;25:249-255.

17. Wu AK, Auerbach AD, Aaronson DS. National incidence and outcomes of postoperative urinary retention in the Surgical Care Improvement Project. Am J Surg. 2012;204:167-171.

18. Hubner M, Blanc C, Roulin D, Winiker M, Gander S, Demartines N. Randomized clinical trial on epidural versus patient-controlled analgesia for laparoscopic colorectal surgery within an enhanced recovery pathway. Ann Surg. 2015;261:648-653.

19. Stubbs BM, Badcock KJ, Hyams C, Rizal FE, Warren S, Francis D. A prospective study of early removal of the urethral catheter after colorectal surgery in patients having epidural analgesia as part of the Enhanced Recovery after Surgery programme. Colorectal Dis. 2013;15:733-736. 
20. Coyle D, Joyce KM, Garvin JT, et al. Early post-operative removal of urethral catheter in patients undergoing colorectal surgery with epidural analgesia-a prospective pilot clinical study. Int J Surg. 2015;16:94-98.

21. Hu Y, Craig SJ, Rowlingson JC, et al. Early removal of urinary catheter after surgery requiring thoracic epidural: a prospective trial. J Cardiothorac Vasc Anesth. 2014;28:1302-1306.

22. Kranke P, Jokinen J, Pace NL, et al. Continuous intravenous perioperative lidocaine infusion for postoperative pain and recovery. Cochrane Database Syst Reu. 2015;7:CD009642.

23. Baeriswyl M, Kirkham KR, Kern C, Albrecht E. The analgesic efficacy of ultrasound-guided transversus abdominis plane block in adult patients: a meta-analysis. Anesth Analg. 2015;121:1640-1654.

24. Meylan N, Elia N, Lysakowski C, Tramer MR. Benefit and risk of intrathecal morphine without local anaesthetic in patients undergoing major surgery: meta-analysis of randomized trials. Br J Anaesth. 2009;102:156-167.

25. Motamed C, Farhat F, Remerand F, Stephanazzi J, Laplanche A, Jayr C. An analysis of postoperative epidural analgesia failure by computed tomography epidurography. Anesth Analg. 2006;103:1026-1032.

26. Rigg JR, Jamrozik K, Myles PS, et al. Epidural anaesthesia and analgesia and outcome of major surgery: a randomised trial. Lancet. 2002;359:1276-1282. 